\title{
Smart Robotics for Smart Healthcare
}

\author{
Ritika Wason ${ }^{1}$, Vishal Jain ${ }^{1}$, Gagandeep Singh Narula ${ }^{1 *}$, Anupam Balyan ${ }^{1}$ and Mandeep Kaur ${ }^{2}$ \\ ${ }^{1}$ Associate Professor, Bharati Vidyapeeth's Institute of Computer Applications and Management (BVICAM), New Delhi, India \\ ${ }^{2}$ Associate Professor, School of Engineering \& Technology, Sharda University, Gr. Noida, India
}

*Corresponding author: Gagandeep Singh Narula, Associate Professor, Bharati Vidyapeeth's Institute of Computer Applications and Management (BVICAM), New Delhi, India

\begin{abstract}
The technological advances in ICT, IoT and robotics have enabled development of specific, optimal service-based solutions for many societal issues and problems. Notably in the recent times smart, service-enabled robotics are being applied to impart smart healthcare solutions in healthcare domains like complex surgeries, elderly care, assistive technologies, patient care at home to name a few. This paper is devoted to highlighting how smart robotics is being and can be applied for delivering smart healthcare in varied healthcare perspectives and domain.
\end{abstract}

Keywords: Intelligent Robotics; Smart robotics; Healthcare; Assistive Technologies

\section{Introduction}

Robotics in healthcare holds the potential to aid a number of practices and tasks[1]. Robots can be used to aid people with cognitive, sensory and motor impairments [2]. They can also act as caregivers to ill, injured or elderly people [3]. They are also being utilized in performing complex surgeries in a trained manner [4]. Since long they have also been used to go inside the human body and scan for varied ailments etc. Robots are physically embodied machines that allow precise and real-time movement of instruments and perform functions in an accurate manner eradicating chances of human error [2]. Advancements in technology especially the proliferation of IoT based solutions has transformed a normal human life into smart living through creation of smart environments, smart homes and smart cities [5,6]. Advancements in medical sciences have further created avenues for smart healthcare solutions [7]. This manuscript gives a brief overview of how robotics is been used to enable smart healthcare to improve human life.

\section{Advances in Healthcare Robotics}

Robotics in the present times has proliferated by leaps and bounds delivering successful, precise services and solutions in a number of domains [2]. Robotics with IoT has even transformed healthcare and opened numerous avenues. Some of its key focus areas include: surgical and interventional robotics, rehabilitative robotics, clinical workforce support, elderly and people with disability care, assistive living etc [1], [7-10]. Human Surgery has typically witnessed phenomenal changes with the introduction of robotic surgery [2]. Robots are being successfully applied for a number of surgical operations as depicted in Figure 1 below:

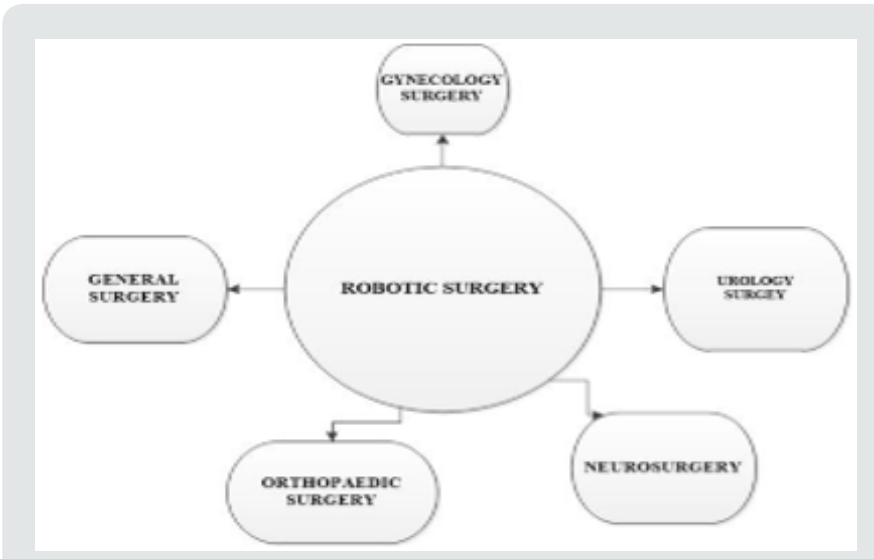

Figure 1: Types of Robotic Surgery. 
Figure 1 above highlights the different avenues where robots have guided and performed minimally invasive surgeries [2]. Intuitive Surgical da Vinci robot has reportedly performed successful surgeries on almost 3 million patients worldwide [4]. They are being successfully used in neurosurgery to examine brain lesions without causing any major damage to the adjacent tissue. In orthopaedic surgeries robots shape the femur to fit prosthetic hip joint replacement with accuracy. With increased life expectancy due to better standards of living a growing demand has unfolded for assistive technologies, healthcare facilities and professionals to provide simple, affordable healthcare services to elderly people or people with disability in an unobtrusive manner. An IoT enabled solution to this demand has emerged in the form of smart homes equipped with wearable medical sensors, actuators and modern IT solutions [5]. ORCATECH Life Laboratory is one such in-house health monitoring solution [2]. Another requirement of trained health professional to provide look after and care to such patients has also been substituted by care bots to provide companionship or look after to patients [11].

\section{Challenges in Healthcare Robotics Adoption}

The advances in healthcare robotics are promising. However it is essential to still note some challenges in the domain and discuss how to overcome them. First and most importantly safety and reliability are critical to healthcare. There need to be efforts to ensure safe and gentle human-robot interactions. This especially needs to be taken care of when patients involved may be people with cognitive disabilities of kids. The next major challenge can be attributed to usability and acceptability of robots by clinicians as well as end users. This demands appropriate technical awareness and training. Only effective awareness, knowledge and training of all stakeholders involved in effective usage of healthcare robots can ensure successful usage of these robots. Another major limiting factor can be cost which includes their purchase, maintenance as well as training costs. Robot manufacturers should aim at devising techniques to manufacture and distribute well-trained and tested robots at the optimal possible costs.

\section{Conclusion}

Healthcare robotics has witnessed promising advances in the recent years. Their usage has also carved many successful stories validating their usage and application. However, how much they will be integrated into the lives of primary beneficiaries still remains unknown as this would be decided by a number of regulating environmental factors which may vary on a case to case basis as the beneficiaries involved are humans.

\section{References}

1. JF Coughlin, LA D’Ambrosio, B Reimer, MR Pratt (2007) Older adult perceptions of smart home technologies: Implications for research, policy \& market innovations in healthcare. Annu Int Conf IEEE Eng Med Biol - Proc pp.1810-1815.

2. Nurgia (2016) Healthcare surgery embraces world of smart robots. Financial Times.

3. LD Riek (2017) Healthcare Robotics 60(11): 68-78.

4. (2018) Surgical Robotics Transforming the Smart Healthcare. Robotics Tomorrow.

5. M Chen, Y Ma, J Song, CF Lai, B Hu (2016) Smart Clothing: Connecting Human with Clouds and Big Data for Sustainable Health Monitoring. Mob Networks Appl 21(5): 825-845.

6. S Majumder, E Aghayi, M Noferesti, H Memarzadeh-Tehran, T Mondal, et al. (2017) Smart Homes for Elderly Healthcare-Recent Advances and Research Challenges. Sensors 17(11): 2496.

7. X Wang, Q Gui, B Liu, Z Jin, Y Chen (2014) Enabling smart personalized healthcare: A hybrid mobile-cloud approach for ECG telemonitoring. IEEE J Biomed Heal Informatics 18 (3): 739-745.

8. M Chan, D Estève, C Escriba, E Campo (2008) A review of smart homesPresent state and future challenges. Comput Methods Programs Biomed 91(1): 55-81.

9. J Li, J Jin, D Yuan, M Palaniswami, K Moessner (2015) EHOPES: Datacentered Fog platform for smart living. $25^{\text {th }}$ Int. Telecommun. Networks Appl Conf ITNAC, pp. 308-313.

10. M Gillham, B McElroy, G Howells, S Kelly, S Spurgeon, et al. (2012) Weightless neural system employing simple sensor data for efficient real-time round-corner, junction and doorway detection for autonomous system path planning in smart robotic assisted healthcare wheelchairs. Proc. - $3^{\text {rd }}$ Int Conf Emerg Secur Technol EST, pp. 161-164.

11. M Chan, E Campo, D Estève, JY Fourniols (2009) Smart homes - Current features and future perspectives. Maturitas 64(2): 90-97.

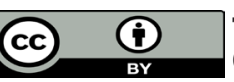

This work is licensed under Creative Commons Attribution 4.0 License

To Submit Your Article Click Here:

Submit Article

DOI: 10.32474/ARME.2019.01.000121

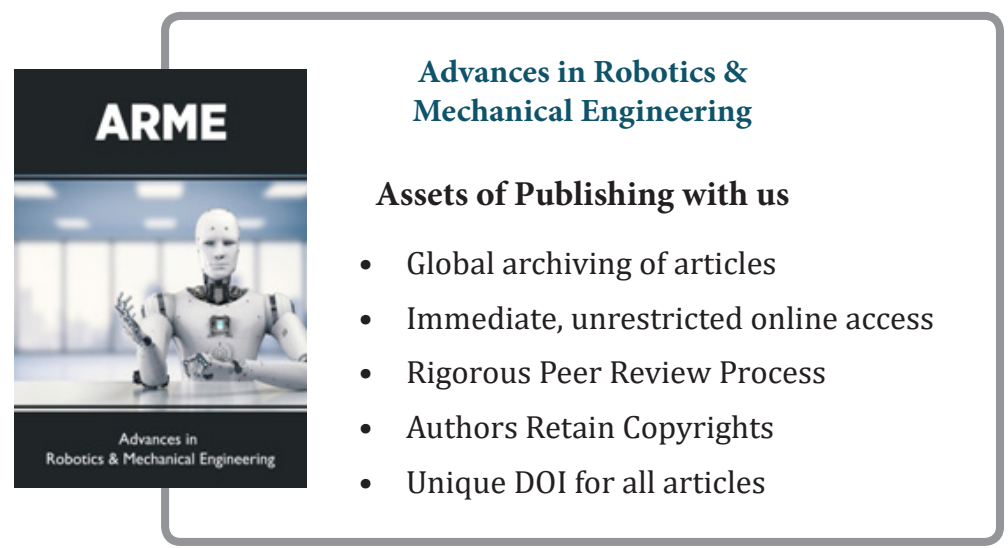

